\title{
CAUSATION, EFFECTUATION, IMPROVISATION ET AGIR ENTREPRENEURIAL. POUR UNE APPROCHE RENOUVELÉE ET INTÉGRATIVE DE L'ENTREPRENEURIAT
}

\author{
Christophe Schmitt, Pierre-André Julien
}

De Boeck Supérieur | «Projectics / Proyéctica / Projectique »

2020/1 n²5 | pages 131 à 153

ISSN 2031-9703

ISBN 9782807393868

Article disponible en ligne à l'adresse :

https://www.cairn.info/revue-projectique-2020-1-page-131.htm

Distribution électronique Cairn.info pour De Boeck Supérieur.

(C) De Boeck Supérieur. Tous droits réservés pour tous pays.

La reproduction ou représentation de cet article, notamment par photocopie, n'est autorisée que dans les limites des conditions générales d'utilisation du site ou, le cas échéant, des conditions générales de la licence souscrite par votre établissement. Toute autre reproduction ou représentation, en tout ou partie, sous quelque forme et de quelque manière que ce soit, est interdite sauf accord préalable et écrit de l'éditeur, en dehors des cas prévus par la législation en vigueur en France. Il est précisé que son stockage dans une base de données est également interdit. 


\section{CAUSATION, EFFECTUATION, IMPROVISATION ET AGIR ENTREPRENEURIAL. POUR UNE APPROCHE RENOUVELÉE ET INTÉGRATIVE DE L'ENTREPRENEURIAT}

\section{Christophe Schmitt}

IAE Metz, CEREFIGE, Université de Lorraine

\section{Pierre-André Julien}

Université du Québec à Trois-Rivières

\section{RÉSUMÉ}

Les travaux portant sur l'effectuation ont considérablement fait évoluer la recherche en entrepreneuriat. Initialement positionnés en opposition aux approches causales, ces travaux ont permis de montrer la présence de la logique effectuale, mais aussi d'autres logiques.
Dans cet article, les auteurs proposent d'envisager ces logiques de fonctionnement à travers l'angle de l'agir entrepreneurial (Schmitt, 2015) qui inclut celles-ci et l'improvisation, mais qui va au-delà pour expliquer les comportements des entrepreneurs à court et à moyen terme. Cette perspective de l'agir entrepreneurial permet non seulement de mieux comprendre ces comportements, mais aussi l'entrepreneuriat régional ou national, la question du temps et les logiques collectives dans la dynamique entrepreneuriale; dans ce dernier cas, en tenant compte, par exemple, des parties prenantes, mais aussi de l'écosystème, de façon à situer et à distribuer cet agir entre plusieurs.

Mots-clés : effectuation, causation, improvisation, agir entrepreneurial, rationalité collective 


\section{ABSTRACT}

The work on effectuation has significantly changed research in entrepreneurship field. Initially positioned in opposition to causal approaches, this work made it possible to show the presence of effectal logic, but also other kinds of logic. In this article, the authors propose to consider these operating logics through the angle of entrepreneurial act (Schmitt, 2015) which includes these logics as much as effectuation, but which aims also to go beyond in order to explain the entrepreneurs behaviors at the short and medium terms. Indeed, the perspective of entrepreneurial act allows not only to better understand these behaviors, but also to apprehend regional or national entrepreneurship, the matter of time and the collective logics in entrepreneurial dynamics. In the latter case, it takes into account, for instance, stakeholders, but also the ecosystem, in order to both locate and distribute the entrepreneurial act among several components.

Keywords: effectuation, causation, improvisation, entrepreneurial act, collective rationality

\section{INTRODUCTION}

Ces dernières années, la focalisation sur de nombreux travaux consacrés à l'effectuation, faisant suite à l'article de Sarasvathy de 2001, a amené une bonne partie de la recherche en entrepreneuriat à se positionner par rapport à l'hypothèse plus ou moins implicite de dichotomie entre la causation et l'effectuation. Les travaux qui suivirent ont permis toutefois d'en arriver à une certaine sophistication au niveau de la compréhension de l'effectuation, mais aussi de montrer que causation et effectuation ne sont que les deux faces de la manière de comprendre la stratégie à court et à moyen terme des petits entrepreneurs, permettant ainsi de sortir de cette dichotomie pour y voir plutôt une complémentarité (Chandler et al., 2011 ; Smolka et al, 2016, Alsos et al., 2016). Complémentarité que l'on peut étendre à une autre façon de faire qu'est l'improvisation, comme on le verra plus loin. À travers cet article, nous nous inscrivons dans cette optique, en faisant l'hypothèse conceptuelle que causation et effectuation, complétées par cette improvisation, ne sont finalement que trois façons différentes de gérer une PME, selon les circonstances ou le bon vouloir des entrepreneurs et, ainsi, d'appliquer ce qu'on appelle l'agir entrepreneurial', en l'occurrence la manière dont les entrepreneurs développent sur un territoire donné leur entreprise, soutenus par leur écosystème.

En d'autres mots, le renforcement des travaux autour de l'effectuation des dernières années (Perry, Chandler et Markova, 2011 ; Jiang et Rüling, 2019), s'ajoutant à d'autres travaux comme ceux sur la cognition entrepreneuriale (Grégoire, Corbett et McMullen, 2011; Grégoire, Cornelissen, Dimov et van Burg, 2015 ; Schmitt et Grégoire, 2018) ou encore ceux relatifs

1. Notion introduite par Schmitt (2015a). 
à l'intersubjectivité en entrepreneuriat (Sarasvathy et Venkataraman, 2011 ; Dew, Grichnik, Mayer-Haug, Read et Brinckmann, 2015, et Schmitt, 2017a) amène la recherche en entrepreneuriat à s'inscrire, comme le rappelle Schmitt (2017b), dans le paradigme de la décision où l'action est le plus souvent envisagée à travers l'hypothèse implicite ${ }^{2}$ que cette action suit la décision, et ce, de façon linéaire, allant de la première à la seconde ${ }^{3}$, pendant même que la décision lou encore, la décision et l'action, sinon l'indécision et l'inaction : Wood. William et Drover, 2017), notamment appréhendée à travers la cognition entrepreneuriale, redevient un des centres de la réflexion en entrepreneuriat ${ }^{4}$. Face à ce renforcement, des réflexions cherchent à proposer un autre regard. C'est notamment le cas des approches interactionnistes où l'importance du processus est mise en évidence (Johannisson, 1995 ; 2011 ; Steyaert, 2007 ; Steyaert et Landström, 2011 ; Moroz et Hindle 2012 ; Schmitt, 2015b et 2017a), ou encore, des approches portant sur l'action située et distribuée (Hutchins, 1995 ; Schmitt, 2009 ; Welter, 2011 ; Dew et al., 2015). C'est dans cette lignée que nous proposons d'inscrire notre analyse sur la relation entre causation et effectuation, en l'étendant toutefois à d'autres pratiques pour gérer les entreprises, telle l'improvisation, et en situant le tout dans une logique plus large qu'est l'agir entrepreneurial sur un territoire donné.

L'objectif de notre réflexion est donc non seulement d'interroger la structuration de la recherche en entrepreneuriat en utilisant la confrontation entre les différentes logiques ou pratiques, mais aussi de reprendre ce questionnement comme tremplin afin de proposer des pistes nouvelles de recherche. Objectif permettant ainsi de mieux asseoir ces approches autour du concept plus large de l'agir entrepreneurial, comme le propose Schmitt (2015a), afin de mieux inclure ces notions de causation, d'effectuation et d'improvisation dans une vision collective et dynamique ou temporelle. Notre réflexion s'inscrit par conséquent dans une perspective renouvelée ou intégrative que nous évoquerons plus loin, en proposant de faire évoluer les cadres habituellement mobilisés dans la recherche en entrepreneuriat.

Pour ce faire, après avoir présenté l'action selon la causation et l'action selon l'effectuation, nous proposons d'envisager ces deux logiques ou pratiques sous l'angle de la complémentarité autour de ce concept avec ses possibilités et ses contraintes, tout en discutant de l'improvisation dans son rapport aux comportements différents des dirigeants de gazelles. Nous terminerons notre analyse en résumant les principaux points de notre argumentation pour ensuite proposer ce qui reste à faire pour mieux tenir compte de l'action collective dans la théorie entrepreneuriale toujours en développement.

2. Sauf dans quelques articles ou livres l'explicitant directement, tels chez Smith et Di Gregorio (2002). McMullen et Sherpherd (2006), Klein (2008), McKelvie, Haynie et Gustafsson (2011), Corbett et Katz (2012), Watson (2013), Frese (2014), ou Lerner, Hunt et Dimov (2018).

3. La recherche en entrepreneuriat, à bien des égards, n'est pas sortie des schémas linéaires mobilisés habituellement par la recherche, comme le schéma behavioriste de stimuli-réponses. Même leur sophistication, avec des boucles de rétroaction, ne permet pas de sortir de la linéarité dans laquelle ces schémas s'inscrivent. Le schéma de l'effectuation proposé par Sarasvathy et al. (2007) n'échappe pas à ce constat.

4. Cette idée n'est pas récente, comme le rappelle Koenig (2003, p. 28) : « nourri aux sources du pragmatisme, Karl Weick rompt avec une manière très ancrée dans notre culture de subordonner l'action à la pensée ». 


\section{LES APPROCHES CAUSALES : DE L'AGIR RATIONNEL À L'AGIR NORMATIF}

Les approches causales ont largement dominé le développement de la recherche en entrepreneuriat. Comme le suggère Schmitt (2015a), il est possible d'envisager le développement de la recherche en entrepreneuriat à travers différents agirs. Ces agirs devraient même devenir le fondement et la structuration de la recherche en entrepreneuriat. Plus précisément, deux questions ont façonné cette recherche et ont ainsi permis le développement de l'agir rationnel (que fait l'entrepreneur ?) et de l'agir normatif lqui est l'entrepreneur ?). Concernant la première question, elle renvoie non seulement à la fonction de l'entrepreneur dans la société, mais aussi aux résultats des actions que ce dernier met en place. Cette place de l'action se retrouve dès les fondements de la pensée économique classique à travers les travaux de Cantillon prolongés par Say. Les rares économistes qui se soient intéressés à l'entrepreneuriat par la suite, comme Schumpeter, Knight ou Kirzner (Klein, 2008), se sont largement inscrits dans cette logique. Ces chercheurs ont contribué à la sophistication dans la pensée économique de cette fonction sociale de l'entrepreneur et de sa rationalité. Ce qui a permis la construction des théories en entrepreneuriat autour d'une abstraction: l'homo œconomicus. Toutefois, des brèches ont vu le jour à travers des travaux comme ceux de Gartner (1988) qui peuvent être considérés comme une première tentative pour ébranler le paradigme dominant de cette approche causale.

Sans forcément s'affranchir d'un agir rationnel de ces économistes traditionnels, les réflexions en entrepreneuriat se sont développées à travers d'autres domaines faisant émerger une posture différente par rapport à l'action. Ce sont les psychologues qui vont fortement imprégner à partir des années soixante-dix la représentation de l'entrepreneuriat autour d'une théorie de l'action centrée essentiellement sur l'individu nécessairement rationnel, selon Max Weber. Plus particulièrement, c'est le courant behavioriste qui viendra s'emparer de la question «qui est l'entrepreneur? ». L'objectif de ces derniers est de comprendre l'entrepreneuriat à travers les aspects observables. Il s'agit d'utiliser des modèles issus de la psychologie sociale et cognitive et touchant les intentions (Ajzen, 1991 ; Boyd et Vozikis, 1994). Les travaux les plus reconnus dans ce domaine renvoient à la connaissance des traits de caractère. Ceux relevant de la théorie du comportement planifié (Shapero, 1975 ; Shapero et Sokol, 1982) sont les plus souvent cités. Ces traits de caractère sont envisagés bien souvent indépendamment du contexte, de l'évolution des entrepreneurs et des mécanismes d'apprentissage. Ainsi l'entrepreneuriat et la réussite entrepreneuriale se comprennent-ils essentiellement à travers l'entrepreneur et ses qualités intrinsèques qui deviennent des références. On parlera dans ces conditions d'agir normatif.

Ces deux approches peuvent être considérées comme réductrices car elles supposent que l'entrepreneur doit être envisagé de façon déconnectée de son environnement. En s'inscrivant dans un certain déterminisme, ces manières de percevoir l'entrepreneuriat ont perdu totalement de vue la liberté de choix 
et de décision, de même que la capacité d'apprentissage qui caractérise l'homo œconomicus.

\section{LES APPROCHES EFFECTUALES : LE RENFORCEMENT DE LA PRISE DE DÉCISION À TRAVERS L'AGIR COGNITIF}

Devant les limites de l'approche causale, un nouvel agir, en l'occurrence l'agir cognitif, prend forme pour aborder sous un angle différent l'entrepreneuriat dont l'objectif se situe essentiellement au niveau de la prise de décision (Schmitt, 2015a). Ce sont en particulier les travaux menés par Sarasvathy $12001 ; 2003 ; 2008)$ qui sont à l'origine de cette émergence autour de son concept d'effectuation, menant plus globalement à une nouvelle réflexion sur les pratiques entrepreneuriales (Schmitt, 2015b). Dans cette logique, la question centrale devient : comment l'entrepreneur décide-t-il ? Les principaux agir que sont l'agir rationnel et celui normatif se sont construits autour des questions génériques du «Quoi ?» et du «Qui ? ». L'agir cognitif, pour sa part, amène une tout autre question générique autour du «Comment?». Il ne s'agit plus de savoir ce que nous connaissons à partir du «Quoi ? » et du «Qui ? », mais plutôt de savoir comment nous connaissons l'entrepreneur, pour reprendre la pensée de Von Foerster (2000), quels sont ses mécanismes cognitifs, mais aussi et surtout quelles sont ses pratiques avec son organisation et en tenant compte de son environnement et de ce qu'il en connaît ou en imagine ${ }^{5}$. Ce glissement n'est pas anodin, et les implications de ce changement de perspective sont importantes. Avec ces travaux, la recherche se déplace d'une compréhension de la réalité par le chercheur vers une appréhension des mécanismes pour aborder la réalité de l'entrepreneur.

Ainsi, au lieu de considérer que les moyens mis en œuvre, quels qu'ils soient, convergent vers un même but, Sarasvathy (2008) soutient-elle qu'un ensemble de moyens donnés peut permettre d'atteindre des buts différents pas forcément connus a priori, mais relevant jusqu'à un certain point de l'anticipation. À partir d'un travail effectué auprès d'entrepreneurs qui ont réussi dans des moyennes entreprises, Sarasvathy montre que ce type d'entrepreneurs se focalise plus sur les possibilités de l'utilisation des ressources existantes dans l'entreprise ou accessibles dans l'écosystème comme instrument pour définir graduellement leurs buts. Là encore, des liens peuvent être établis avec la théorie des ressources élaborée par Penrose (1959) et reprise dans l'approche des ressources et compétences (Wernerfeld, 1995 ; Marchesnay, 2002), qui considère celles-ci comme une source de création de valeur devant être « achetées » par le marché, sans toutefois en être assurée. Les réflexions portant sur l'opportunité symbolisent bien l'apport de l'effectuation ${ }^{6}$ au niveau

5. Von Foerster affirme que la question du « quoi » se veut essentiellement explicative à la différence de la question du « comment » qui, elle, se veut essentiellement compréhensive.

6. Rappelons que ce concept d'effectuation se retrouve dans les travaux de 1860 du philosophe Charles Sanders Peirce et a été repris par Gilles Deleuze et Félix Guattari dans Milles Plateaux de 1980. 
de la recherche en entrepreneuriat. L'effectuation, notamment par rapport à cette notion d'opportunité, cherche à comprendre les mécanismes de réflexion de l'entrepreneur comme individu. La rationalité, vue ici en tant que processus de prise de décision, est donc aussi présente même si elle évolue, car elle est avant tout considérée comme procédurale, selon l'acception de Simon (1996).

De même, cette logique effectuale tient compte implicitement d'un autre acteur qui est l'environnement ou l'écosystème et sa représentation par l'entrepreneur (Marchesnay et Julien, 1990). Pendant que dans le discours économique traditionnel avec le capitalisme ambiant et sur le management en général, et l'entrepreneuriat en particulier, l'environnement apparaît comme une arène ouverte où les concurrents s'affrontent et tentent de dégager un avantage concurrentiel, il est proposé de porter un autre regard sur cet écosystème ou sur le contexte (Acs, Desai et Klapper, 2008 ; Henrekson et Johansson, 2010 ; Fritsch et Wyrwich, 2017). Certains autres acteurs de l'entrepreneuriat, en plus de l'entrepreneur, peuvent en effet être envisagés comme des parties prenantes participant directement ou indirectement à la décision, à mesure que la trajectoire de l'action suivie ou corrigée le requiert. L'écosystème joue un second rôle avec l'apport du capital social, complétant ces ressources complémentaires évoquées précédemment, et l'importance du réseautage ajoutant l'information nouvelle lorsque la réalité le réclame pour mieux comprendre le changement au cours de l'avancement du projet, de façon à s'ajuster aux nouveaux besoins et, ainsi, mieux y faire face (Geindre et Dussuc, 2015 ; Razafindrazaka et Julien, 2019). En somme, l'ambition de Sarasvathy (2003) et de Sarasvathy et Venkataraman (2011) est grande : porter un regard différent sur l'entrepreneuriat en considérant aussi ses parties prenantes et cet écosystème dans une logique partenariale.

Il n'en demeure cependant pas moins que cette logique ne s'oppose pas nécessairement à celle causale, comme nous l'avons dit en introduction et comme le rappellent des chercheurs critiques, tels Arend, Sarooghi et Burkemper (2015), mais aussi des analyses appliquées récentes, telles celles de Chandler et al. (2011), Gabrielson et Politis (2011), ou Alsos et al. (2016). En effet, rien n'empêche le recours à l'une et à l'autre des approches, et même de les utiliser simultanément selon les circonstances. Ce que font, par ailleurs, la plupart des entrepreneurs.

D'ailleurs, le choix entre ces deux approches ne relève pas du hasard. Même les entrepreneurs experts de Sarasvathy doivent recourir aussi à des démarches causales. Ce choix s'explique par les différences importantes qui surviennent dans le développement des PME, différences liées à l'importance des projets ou du temps requis pour les appliquer, au niveau d'incertitude ${ }^{7}$ qui environne ces derniers et, enfin, à l'expérience diversifiée de l'entrepreneur et de son organisation.

Ainsi les petits projets suivent-ils généralement une démarche causale orientée par des objectifs relativement précis. Il en est de même pour des

7. A noter que cette incertitude est avant tout subjective (Duncan, 1972; Milliken, 1987), selon l'expérience et le niveau de risque envisagé par l'entrepreneur, mais aussi selon l'apport des parties prenantes dans leur appréciation de cette incertitude, puisqu'elle porte sur le futur qui n'existe pas encore. Ce qui est aussi le cas des opportunités qui relèvent du futur et qui ne peuvent être que subjectives (Klein, 2008). 
dizaines de petites opportunités touchant des ajustements dans le produit ou encore dans les façons de produire. Cette démarche pour les projets mineurs est favorisée du fait que l'incertitude, dans ce cas, est généralement faible ; puisqu'il est facile d'en prévoir les conséquences et d'en anticiper réellement les étapes et les coûts pour y arriver. Cela se fait à l'encontre des grands projets ou des opportunités importantes qui demandent du temps pour se réaliser et dont les résultats ne sont pas clairs et encore moins assurés, compte tenu des comportements des concurrents et des changements fréquents dans l'environnement. Dans ce cas, plus l'incertitude est grande, plus il est préférable d'utiliser une démarche effectuale offrant assez de souplesse pour s'ajuster à l'information obtenue en cours de route, quitte à changer son fusil d'épaule rapidement si les nouvelles conditions l'exigent (McKelvie, Haynie, et Gustavsson, 2011 ; Welter et Kim, 2018).

Enfin, l'expérience de l'entrepreneur et de son organisation, et leurs capacités particulières à imaginer les opportunités (Baron, 2006 ; Klein, 2008 ; Vaghely et Julien, 2010) et à anticiper le changement (Davenport et al., 1998 ; Liao et al., 2008) peuvent favoriser l'approche effectuale plutôt que celle causale. Ainsi est-il probable que la plupart des nouveaux entrepreneurs recourent à l'approche causale ${ }^{8}$, d'autant plus que leurs ressources sont le plus souvent limitées et leurs marges de manœuvre faibles. Ce comportement causal est encore plus fréquent quand la nouvelle entreprise reproduit ou imite d'autres entreprises, comme il arrive souvent quand le nouvel entrepreneur a développé son expérience préalable dans une entreprise qu'il veut imiter avec quelques différences mineures. Ce n'est que plus tard, après avoir acquis de l'expérience, pris confiance dans ses intuitions et amélioré ses capacités à réagir, que l'entrepreneur passe à des actions effectuales. C'est bien ce que Bruyat et Julien (2001) mettent en lumière, en rappelant que l'expérience en création de valeur transforme en même temps l'entrepreneur. Faisant suite à cette évolution, il pourra graduellement tenter des expériences effectuales en s'engageant dans des projets importants requérant temps et ressources, tout en gageant sur sa bonne étoile (Shackle, 1979).

Revenons à la quatrième variable len sus de l'ampleur du projet, de l'expérience de l'entrepreneur et du degré d'incertitude, dont nous venons de parler) qui est la capacité de l'écosystème, dans lequel se développe chaque entreprise, de fournir des ressources complémentaires, tels du personnel ou du nouveau financement, de même que de l'information potentielle par le réseautage, notamment dans le déroulement des projets importants. Le tout demandant du temps et augmentant ainsi l'incertitude (Welter et Kim, 2018). L'action des entrepreneurs ne peut être comprise que de façon dynamique (Lévesque et Stephan, 2019) dans son contexte ou son écosystème et la collectivité qui l'entoure (Hindle, 2010), y compris sa culture et ses conventions (Bruton, Ahlstrom et Li, 2010 ; Estrin, Korosteleva et Mickiewicz. 2013). Cette culture imprègne les façons d'être et les règles de jeu qui dictent en partie les façons de faire des affaires. En d'autres termes, l'action de tout entrepreneur ne peut être bien comprise que dans la connaissance et l'interaction qu'il développe avec son environnement, le tout s'inscrivant dans l'agir entrepreneurial.

8. Mais pas nécessairement, comme l'expliquent Smolka et al. (2016) dans une enquête auprès de 1453 nouveaux entrepreneurs provenant de 25 pays. 


\section{L'AGIR ENTREPRENEURIAL}

Si cette action est un concept clé pour bon nombre de domaines en sciences humaines et sociales (Parsons, 1937 ; Boudon, 1984 ; Joas, 1996), en se focalisant sur la décision et encore plus avec les recherches récentes évoquées précédemment, la recherche en entrepreneuriat s'en est trop souvent trop éloigné. Dans ces disciplines, la conception d'une «théorie de l'action » doit être un élément central de la réflexion. Il s'agit non seulement de s'intéresser au développement d'une telle théorie en entrepreneuriat (McMullen et Shefferd, 2006), mais de façon concomitante de s'interroger encore plus sur la place de l'action dans les pratiques entrepreneuriales. Cette « théorie de l'action » correspond à ce que nous appelons, à travers la proposition de Schmitt (2015a), l'agir entrepreneurial, permettant d'englober les trois pratiques évoquées précédemment. À travers cet agir, il ne s'agit pas de tomber dans une nouvelle dichotomie d'un côté, avec la prise de décision et, de l'autre, l'action entrepreneuriale, mais d'appréhender de façon plus globale cet agir dans lequel l'action et la décision sont concomitantes. Sans compter d'autres éléments, conscients ou non, comme l'émotion, la confiance en soi, l'intuition, et même la passion, pouvant même mener à de l'irrationalité ${ }^{9}$ (Grichnik, Smeja et Welpe, 2010 ; Lerner, Hunt et Dimov, 2018), ou encore la capacité de projection : éléments qui vont se nourrir mutuellement et non de façon linéaire. Plus précisément, notre discussion se place principalement sur le terrain des liens entre connaissance et action et, par conséquent, sur le plan de l'agir. Ce projet d'une meilleure prise en compte de l'action ou d'une meilleure articulation avec la décision paraît aujourd'hui essentiel si nous souhaitons faire avancer la recherche en sortant encore plus des sentiers battus. Il est d'autant plus important qu'il met en jeu l'orientation du domaine entrepreneurial de recherche tout entier et sa ligne de démarcation par rapport aux autres domaines.

L'agir entrepreneurial pourrait se définir par des actions et des décisions dans lesquelles chaque entrepreneur est en interaction avec ses parties prenantes et les autres acteurs, dont les autres entreprises, dans son écosystème, et ce, dans le temps ou de façon dynamique (Schmitt, 2015a). L'entrepreneur évolue de façon adaptative et en partie volontaire avec cet écosystème qu'il construit à partir de ses représentations de la situation réelle dans laquelle il se trouve (Marchesnay et Julien, 1990). Ces représentations mettent ainsi en relation l'entrepreneur avec son contexte à partir d'un futur souhaité et en fonction de son intentionnalité ${ }^{10}$, de ses moyens ou ressources et de ses fins générales et spécifiques. Cette conception considère «l'agir entrepreneurial comme un construit humain finalisé à travers les représentations de l'entrepreneur et des autres acteurs » (Schmitt, 2015a). Cet agir est vu ainsi comme une construction ciblée de l'entrepreneur en relation avec cet écosystème (personnes et artefacts) par ses actions et ses décisions (Schmitt, 2017b).

9. Surtout quand l'entrepreneur se situe dans un environnement très incertain alors qu'il doit agir, ne serait-ce que pour que son organisation «avance ». D'autant plus qu'il doit décider avec trop peu de temps pour réfléchir.

10. L'intentionnalité désigne la capacité qu'a un individu de se forger des représentations portant son empreinte (désir, volonté, rapport au monde...) à l'instar des actions qu'il entreprend. 
Cette relation à autrui et aux artefacts est importante puisque c'est elle qui, en ayant mesuré l'importance du projet en particulier et le niveau d'incertitude dans lequel il baigne, et selon son niveau d'expérience, conditionne en quelque sorte le choix entre une approche causale et une approche effectuale, sinon d'improvisation. Selon Schmitt (2015a et b), comme toute personne, l'entrepreneur ne peut se dissocier ni de lui-même, ni de son organisation, ni de son action en situation, encore moins de ses effets sur les autres entreprises, les autres acteurs et même sur l'économie en général et la concurrence, bref sur les autres actions et acteurs (Davidsson, 2015 ; Fritsch et Changoluisa, 2017). L'entrepreneur et l'action participent au développement de l'agir de celui-là par adaptation de son intentionnalité et de ses actions tant causales qu'effectuales à ses différents environnements. L'agir entrepreneurial apparaît comme un concept permettant de mieux saisir et donc de mieux accéder à l'ensemble du phénomène entrepreneurial. Les actions et les interactions en situation jouent conséquemment un rôle important dans cette compréhension (Venkataraman et al., 2012). L'agir devient dans ce sillage même une nouvelle grille de lecture de l'entrepreneuriat où l'action entrepreneuriale joue un rôle-clé.

Du point de vue temporel, la particularité de l'agir entrepreneurial est le lien tissé entre la situation future souhaitée et la situation actuelle, comme le rappelle Schmitt (2015b). Ce lien entre ces deux moments renvoie à la notion de conception développée par Simon (1996, p. 201), grâce à laquelle ce dernier explique que concevoir correspond à la capacité d'imaginer des dispositions visant à changer une situation existante en une situation préférée et dont la capacité d'imaginer est le point de départ. À travers la relation entre le présent et le futur, cet agir est envisagé comme l'ensemble des circonstances dans lesquelles l'entrepreneur se trouve, mais aussi des desseins de l'entrepreneur à travers cette intentionnalité ${ }^{11}$.

Toutefois, trop souvent les travaux portant sur l'entrepreneuriat s'inscrivent essentiellement dans une temporalité linéaire et conventionnelle du temps, allant du passé au futur en passant par le présent, selon la logique wébérienne : logique d'une « action atomistique, relevant de relations moyens-fins [...] d'un acteur isolé [...] poursuivant ses intérêts personnels [...] négligeant les mécanismes de coordination par lesquels adviennent des relations interpersonnelles [...] et présupposant [...] un monde limité aux états de choses existants », comme l'explique Habermas (1987, p. 282-83). Ce que nous retrouvons bien souvent dans les études de marché, dans les projections financières ou plus généralement dans le plan d'affaires. Cela renvoie à ce que Sarasvathy (2001) désigne par les logiques divinatoires. Étrangement, à écouter les entrepreneurs, on voit qu'il existe une autre relation au temps ${ }^{12}$ que la recherche a du mal à prendre en compte (Lévesque et Stephan, 2019) : le temps va du futur au présent dans une perspective itérative (Schmitt, 2015a). C'est dans l'idée et, plus clairement encore, dans l'opportunité d'affaires, et donc dans

11. Il convient de ne pas confondre intentionnalité avec l'intention entrepreneuriale qui renvoie à une approche behavioriste de l'entrepreneuriat où le comportement de l'entrepreneur peut être prédit (Shapero et Soko, 1982 ; Krueger et Carsrud, 1993), comportement relevant de l'agir normatif.

12. Ou plutôt la durée, rappelant que le temps est subjectif pour tout individu, tels les entrepreneurs, comme l'explique Bergson (1922). 
la projection d'un futur souhaité, que l'on peut comprendre l'action et l'intentionnalité de l'entrepreneur. Il est donc possible d'avancer «que ce n'est pas le passé, mais le futur, qui détermine le présent » (Watzlawick, 2000, p.74). C'est le cas en particulier des gazelles dans lesquelles le futur semble tirer ou, tout simplement, orienter systématiquement le présent. Et, dans ce cas, nous ne sommes même pas dans une linéarité inversée où le futur fait évoluer le présent, mais dans une interaction permettant aussi au présent en retour d'influencer le futur envisagé, notamment les actions mises en place.

Dans cette perspective, l'agir entrepreneurial peut être considéré « comme la mise en ordre et l'organisation d'un monde constitué par nos expériences » (Glasersfeld, 1984, p. 41). Par conséquent la problématique de recherche doit évoluer pour mieux se porter sur l'agir entrepreneurial par le truchement de l'action de l'entrepreneur relative à son rapport au monde (son intentionnalité) dans une perspective phénoménologique de construction de sens, au sens de Weick (1999), ou de construction de problème, au sens de Dewey (1938). Cet agir apparaît alors comme un processus néguentropique (Schmitt, 2017a). Cela signifie qu'il n'y a pas de sens a priori et que ce sens est construit par l'entrepreneur à partir d'une situation existante. C'est ce que nous pouvons retrouver à travers l'approche effectuale, où l'entrepreneur se trouve dans une stratégie « chemin faisant », et encore plus, dans l'approche d'une certaine improvisation que nous expliquons plus largement dans la section suivante. Ainsi, si la logique de résolution de problèmes prévaut dans une approche rationnelle de l'entrepreneuriat, c'est bien une logique de problématisation qu'il convient aussi de prendre en compte pour aborder l'entrepreneuriat (Schmitt, 2015a, 2017a, 2017b). Toutefois, résolution de problèmes et problématisation ne renvoient pas aux mêmes démarches ni aux mêmes outils. Si les démarches et les outils de la résolution de problèmes sont bien connus, il est aussi important de s'intéresser aux démarches et aux outils de la problématisation. En effet, problématiser ${ }^{13}$ nécessite une réelle prise de conscience de l'entrepreneur, de son écosystème, de son projet et de sa personne propre.

\section{POUR ALLER PLUS LOIN : CAUSATION, EFFECTUATION ET IMPROVISATION}

Cette démarche avec une nouvelle logique nous permet d'aller quelque peu plus loin que les approches causales et effectuales en nous arrêtant aux actions des entrepreneurs de PME à forte croissance, ou gazelles ${ }^{14}$, premières créatrices d'emplois dans nos économies (Acs et Mueller, 2008 ; Mitusch et Schimke, 2011 ; Chanut-Guieu et Guieu, 2011), malgré leur importance relativement marginale (moins de $10 \%$, selon l'OCDE, 2002). La plus grande partie

13. Sur ce point, voir les travaux de Schmitt (2015, 2016, 2017).

14. Pour l'OCDE (2002), les gazelles sont des PME de plus de 10 employés qui doublent leur taille à chaque année durant au moins cinq ans. 
du comportement de ces entreprises repose sur l'improvisation, soit une série d'actions orientées mais non planifiées et adaptées à chaque situation (Barrett, 1998 ; Brown et Eisenhardt, 1998), permettant à leur organisation de réagir rapidement et d'assumer ainsi le changement rapide, tant à l'externe qu'en interne. Leurs principales caractéristiques sont, d'abord, une forte proximité avec le marché ou avec leurs clients pour comprendre et se moduler rapidement à leurs besoins (Kim et Mauborgne, 1997), ensuite, un leadership particulièrement fort sachant rappeler systématiquement la direction à suivre afin de conserver la cohésion de l'organisation et de poursuivre les buts de long terme (Hambrick et Croizer, 1985 ; Belliato et al. 2010 ; Chanut-Guieu et Guieu, 2011), puis, une culture organisationnelle participative reposant sur le partage systématique de l'information (Barringer, Jones et Lewis, 1998 ; Grimand, 2013), et enfin, un apprentissage systématique et en bonne partie sur le tas (Cope, 2005). Ainsi plusieurs de ces entrepreneurs que nous avons étudiés (Julien, 2001 ; 2003) affrontent-ils le marché à partir d'une approche carrément prospective tout en ayant recours à leurs réseaux, notamment à signaux faibles (Julien et Lachance, 2001), et en développant des scenarii le plus souvent virtuels et souples ${ }^{15}$. Et ce, soit pour poursuivre la même trajectoire de développement des derniers mois lorsque le potentiel du marché demeure toujours ouvert, ou encore pour imaginer de nouvelles opportunités et réorienter ainsi cette trajectoire, tout en dirigeant globalement l'action de l'entreprise. Pendant que leur organisation continue d'improviser et d'évoluer pour appliquer ces opportunités tant du côté des ressources que de celui du marché, en se pliant systématiquement à ces changements à l'externe tout en ajustant l'organisation en interne - improvisation oblige - vu l'arrivée plus ou moins régulière de nouveaux personnels et d'équipements. Puisque ces entrepreneurs sont occupés à penser l'avenir, au travers de nouveaux liens avec le marché expliqué par cette forte croissance (Barringer, Jones et Lewis, 1998) et avec l'écosystème (Spigel, 2015), ces scenarii les aident à imaginer rapidement l'évolution du marché sinon de nouveaux marchés, mais aussi de nouvelles ressources ou la transformation de celles en place. Ils leur permettent tout autant d'analyser les actions et réactions possibles de la concurrence, pour mieux en discuter, par exemple, avec les parties prenantes, en recourant de la sorte à l'idéation (Vygotsky, 1978), profitant au besoin de leurs encouragements et de leurs conseils (Tang, Tang et Katz, 2014) afin de corriger ces scenarii ou de mieux les développer. Cette façon de faire va au-delà de l'approche de Sarasvathy en considérant les ressources, notamment les employés, comme flexibles et relativement disponibles dans l'écosystème, en fonction de ce qui est possible et souhaitable pour jouer avec le marché (Gjerløv-Juel et Guenther, 2019). Marché, lui-même perçu, dans ce sillage, comme un terrain de jeu plein de potentiels à développer. En d'autres mots, l'improvisation ici porte sur toute l'entreprise, ses stratégies, sa trajectoire, son marché actuel et à venir et son fonctionnement (Smallbone, Leig et North, 1995). Ce faisant, ces entrepreneurs de gazelles représentent la dernière tranche dans les catégories de Bruyat et Julien (2001), soit une partie des entrepreneurs d'aventure. Partie que l'on peut comprendre comme de

15. Ou encore non structurés, à l'encontre des scenarii développés et relativement consistants par certains des autres entrepreneurs (Steyaert, 2007). 
véritables prospecteurs ${ }^{16}$, dont certains affrontent directement le marché en le révolutionnant, répondant ainsi à l'image de Schumpeter, première mouture, où l'entrepreneur transforme jusqu'à un certain point l'économie.

Ajoutons que si ces gazelles sont relativement moins nombreuses que les autres firmes, cela ne veut pas dire que cette improvisation leur est réservée. Il arrive souvent que des PME soient prises avec des urgences pour devoir trouver des réponses rapides, comme s'assurer d'une nouvelle clientèle ou d'une opportunité à saisir très rapidement, les obligeant à recourir dans ce cas à cette logique, même si ce n'est pas courant, tout en corrigeant l'ensemble avec plus de temps quand celui-ci se présente par la suite.

Ajoutons qu'improvisation et, évidemment, causalité et effectuation se distinguent fortement du bricolage, à l'encontre de ce qu'en dit Fisher (2012). Puisque ces trois pratiques, comme dans le cas du jazz d'improvisation, et ce, pour ne pas en arriver à de la cacophonie (Barrett et Peplowski, 1998), supposent une logique, un fil conducteur, ou une trame développée par la direction, dont c'est un des rôles fondamentaux, afin de donner la direction nécessaire pour l'efficience de l'entreprise. Expliquant justement la trajectoire qui peut évidemment se réaliser pour un temps ou consécutivement de façon causale, effectuale ou improvisée selon les opportunités ou les projets plus ou moins importants qui se présentent, comme nous venons de l'expliquer. Alors que le bricolage se limite le plus souvent à des mini-actions dans un temps court, ou encore, avec souvent peu ou pas de liens importants entre les différents bricolages autres que les besoins immédiats complétant les routines et les ajustements nécessaires (Stinchfield, Nelson et Wood, 2012).

La figure suivante résume les trois pratiques ou types d'actions discutées et leur importance dans l'agir entrepreneurial, soit la causation, l'effectuation et l'improvisation, selon les trois variables contraignantes ou potentielles: 1) importance du ou des projets, 2) expérience de l'entrepreneur et de son organisation et 3) incertitude. Sachant que dans le cas des gazelles, le projet global est d'autant plus important qu'il constitue le fil conducteur expliquant la trajectoire même de l'entreprise. Trajectoire relevant justement d'une projection (comme le mot « projet » le suggère), d'une expérience particulière lestimée à plus de 20 ans dans le secteur pour les gazelles : Julien, 2001), et à la grande incertitude assumée. En effet, étant donné que ces entrepreneurs s'engagent dans des sentiers rarement tracés d'avance, la survie de leur entreprise est souvent fragile. Puisque cette forte croissance suppose des investissements continuellement renouvelés, alors que le retour sur ces investissements se fait souvent attendre (St-Jean, Julien et Audet, 2008 ; Claveau, Perez et PrimAllaz, 2013 ; Rannikko et al., 2019).

À noter que ces trois cercles sont partiellement interconnectés, rappelant, comme nous l'avons expliqué, qu'ils doivent être vus en complémentarité et selon une certaine continuité entre les trois types. En d'autres mots, il est probable que la plupart des entrepreneurs, quelles que soient l'importance du

16. Pour reprendre le terme de Miles et Snow (1978), mais en recourant à son sens plus profond. Dans leur cas, les prospecteurs sont de faibles aventuriers, qui peuvent avoir peur du futur, à l'encontre des chercheurs d'or, par exemple, qui laissaient tout tomber, famille, travail, et même pays, pour agir presque comme dans un rêve fou, à moins d'y être plus ou moins obligés, comme dans plusieurs régions très pauvres. 
projet, leur expérience et leur perception de l'incertitude, recourent plus ou moins selon les circonstances à des projets orientés et plus ou moins planifiés et donc causaux, à une prise en compte des capacités des ressources et de leur évolution et, enfin, à une certaine improvisation (Balachandra, 2019). D'autant plus qu'il n'y a pas de frontières strictes, comme toute réalité complexe, entre ces pratiques selon l'évolution des savoirs et des expériences. Évidemment, ces recours à différentes pratiques n'ont ni la même ampleur ni la même fréquence dans chaque entreprise et selon leur histoire. Ce qui nous permet de distinguer ces trois groupes selon le type de pratique, de l'importance du ou des projets, de l'expérience de l'entrepreneur et de son organisation, et de l'incertitude. Tout en tenant compte de la nouvelle valeur créée (Bruyat et Julien (2001) et de leurs objectifs à plus long terme (Wicklund, Davidsson et Delmar, 2003). Enfin, tout cela se fait, 4) en relation ou à l'intérieur de l'écosystème et ses capacités de soutien. Ce qui constitue dans son ensemble l'agir entrepreneurial, c'est-à-dire « le comment » les entreprises agissent selon ces trois pratiques ${ }^{17}$, comme la figure le suggère.

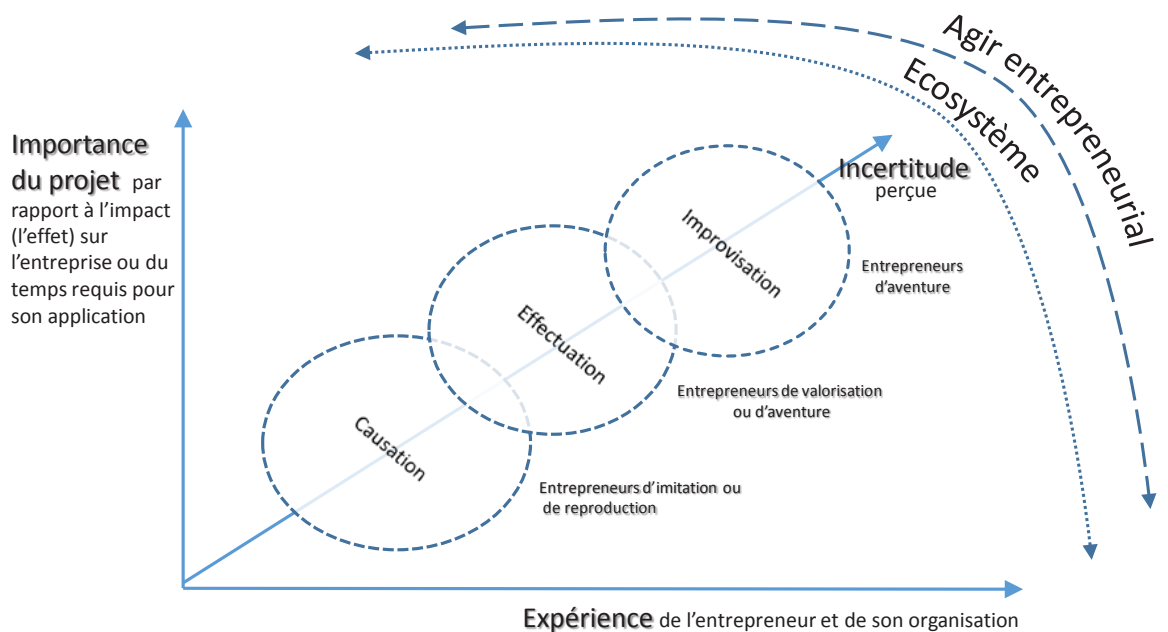

Figure 1. Les 3 pratiques retenues par les entrepreneurs, selon l'importance du projet, l'expérience acquise et l'incertitude perçue, pour leur agir, conditionnées ou aidées par les ressources disponibles dans l'écosystème

En d'autres mots, le troisième type d'entrepreneurs, ceux de gazelles, sont des joueurs qui font fi du moment présent, si l'on peut dire, utilisant le futur pour transformer le futur-présent en vu d'être les premiers à le former, une fois l'information obtenue et transformée. Tout comme le joueur dont parlait Cotta (1980), à l'instar des grands joueurs de football qui improvisent ou inventent sur le tas, par exemple, une nouvelle feinte liée à une stratégie imaginée et bon pour les trois ou quatre coups suivants, forçant les autres joueurs tant de leur camp que chez les adversaires à réagir, si

17. Et ce, même dans un écosystème avec peu de ressources, comme l'expliquent Ngoasong et Kimbu (2019) dans le cas de petites entreprises africaines de ventes de nourriture dans la rue, entreprises à forte croissance dirigées par des entrepreneures. 
possible, en improvisant eux-mêmes et en essayant d'appréhender les prochains coups que ce grand joueur fera. On sait toutefois que cette invention n'est pas constante et requiert des pauses pour consolider leur organisation et le marché en cours (Godemer, 2002 ; St-Jean, Julien et Audet, 2008). Nous savons que ces entrepreneurs-joueurs refusent les routines, préférant au besoin laisser la gouvernance de leur entreprise au profit d'une autre aventure avec de nouveaux défis, comme nous l'avons vérifié à plus d'une reprise (Julien, 2005, p. 85-93).

\section{DES PISTES DE RECHERCHE POUR MIEUX ABORDER L'AGIR ENTREPRENEURIAL}

L'ethnométhodologie (Garfinkel, 1967) permet d'aller encore plus loin dans la compréhension de la notion de l'agir entrepreneurial. Elle met en avant son aspect situé et distribué ${ }^{18}$, dont nous avons parlé plus haut, à travers l'action. Les chercheurs ayant travaillé dans cette perspective considèrent que l'agir n'est que très rarement clairement défini (Blumer, 1969 ; Berger et Luckmann, 1966 ; Suchman, 1987), comme on peut le comprendre dans le dernier cas de l'improvisation. Cet agir s'inscrit dans une théorie relationnelle et dépendante des circonstances de l'activité (Goffman, 1974) que l'on retrouve au niveau de l'entrepreneuriat à travers les travaux portant sur les approches interactionnistes (Johannisson, 1995 ; 2011 ; Steyaert, 2007 ; Steyaert et Landström, 2011 ; Moroz et Hindle, 2012, Schmitt, 2015a et 2017a), et que nous avons cités en introduction. Le qualificatif « situé » suppose que les actions et leur contexte sont uniques, changeants et fugaces, et surtout liés aux représentations de chaque entrepreneur et de son histoire. Ce qualificatif prend ainsi en compte l'écosystème de l'entrepreneur sans lui assigner nécessairement de rôle en dehors d'une relation explicite afin d'obtenir l'engagement des intervenants dans ce dernier pour chaque entreprise (Read et Sarasvathy, 2005) et afin de fournir les ressources complémentaires nécessaires pour soutenir les projets. Ainsi les entrepreneurs traitent-ils les situations en fonction de ce qu'ils pensent qu'elles sont et du sens subjectif qu'elles ont pour eux (Schmitt, 2009), engendrant des logiques causales, effectuales ou d'improvisation. Il convient donc de pouvoir comprendre et analyser les situations dans lesquelles l'entrepreneur se trouve et l'expérience qu'il a en situation (Marchesnay et Julien, 1990).

Du point de vue méthodologique, par ailleurs, il est nécessaire pour le chercheur de ne pas se limiter à des données a posteriori qui ne sont que

\footnotetext{
18. Sur ce point, nous nous opposons toutefois à Dew et al. (2015) qui considèrent la dimension distribuée comme un élément de la dimension située de l'action. Nous préférons parler d'action entrepreneuriale qui serait notamment composée de la dimension située et de la dimension distribuée, comme la littérature dans le domaine de la cognition le montre (Lave, 1988).
} 
déclaratives ${ }^{19}$, comme c'est souvent le cas en entrepreneuriat pour mobiliser des données en situation (Schmitt, 2017b). Des protocoles méthodologiques qui ont fait leurs preuves existent déjà, qu'il conviendrait bien de mobiliser dans ce domaine de l'entrepreneuriat ${ }^{20}$. Le sens n'est pas préétabli dans l'action, ni dans la décision et encore moins dans l'écosystème (Schmitt, 2015a et b). Bien au contraire, le sens est à faire advenir par le chercheur auprès de l'entrepreneur par rapport à son expérience en situation, qu'il soit dans une logique causale, effectuale ou d'improvisation. Le rôle du chercheur évolue afin d'appréhender la manière dont les entrepreneurs utilisent leur agir pour effectuer une action intelligente selon leur expérience. Ainsi l'idée de planification en entrepreneuriat perd-elle de sa pertinence. Pour sa part, l'agir entrepreneurial requiert tout autant cette stratégie chemin faisant en recherche, bien que celle-ci soit d'importance relative sinon très partielle selon la robustesse du projet, l'expérience de l'entrepreneur et de son organisation, et le poids de l'incertitude, comme nous l'avons expliqué en supra.

De son côté, le développement de l'action distribuée (Hutchins, 1995) ou coordonnée ${ }^{21}$, c'est-à-dire partagée et organisée entre plusieurs, met en lumière le fait que des individus, travaillant ainsi en interaction, et donc, en intercommunication et en intercompréhension (Habermas, 1979, p. 291-93), sont susceptibles d'avoir des connaissances différentes et auraient tendance à agir différemment si cette intercompréhension ne se développait pas. Ce qui veut dire que tout entrepreneuriat est jusqu'à un certain point collectif, à l'encontre de la théorie traditionnelle où cela relevait de l'individu, l'entrepreneur, notamment le héros schumpétérien (Bird et Schjoedt, 2009). Pour les tenants de la théorie de l'action, nous serions ainsi dans l'agir entrepreneurial dès que deux ou plusieurs individus se trouvent liés de façon mutuelle et immédiate, sans toutefois être physiquement coprésents. Comme nous pouvons le constater à tout moment, l'agir entrepreneurial est relatif aussi à l'apport et à la qualité d'individus nommés parties prenantes (famille, amis, fournisseurs, clients, salariés, familles, financeurs...), y compris dès la création (Lerner, 2016) ; même si dans ce cas, l'intervention de ces parties prenantes est généralement limitée à cause du manque d'expérience du créateur. Ainsi l'agir entrepreneurial se construit-il à partir du moment où l'entrepreneur prend en compte son action et ce qu'en ressent son ou ses clients, ses financeurs potentiels, en tout cas toute personne qui serait en lien ou comme participant passivement ou activement à son projet, en plus de ses réseaux, le tout évoluant dans le temps (Kerr et Coviello, 2019). Il s'agit d'y voir l'apport de l'action distribuée. Celle-ci vient parachever les travaux sur la vision entrepreneuriale en introduisant la nécessité de traduire celle-ci auprès de ces parties

19. Nous entendons ici par « déclaratif » le fait que le chercheur se limite aux discours de l'entrepreneur ou des acteurs de l'écosystème en les considérant comme une vérité objective sans tenir compte des représentations ni de la construction de ces représentations de l'entrepreneur ou des acteurs de l'écosystème. Sachant que dans toute « enquête », le répondant tend à essayer de «faire plaisir » à l'intervieweur et, ainsi, à ajuster quelque peu ses réponses à ce qu'il comprend des questions.

20. Nous pensons notamment au processus de construction identitaire (Dubar, 1996), à l'auto-confrontation (Theureau, 1992), ou à l'entretien d'explicitation (Vermersch, 1994), permettant des analyses de conversation et d'actions effectuées.

21. Habermas (1981, p. 185) explique que toute action sociale, telle l'entrepreneuriat ici, relève « d'un agir coordonné qui produit dans la société une demande déterminée de communication, qui doit être satisfaite si une coordination effective des actions est possible afin de satisfaire les besoins ». 
prenantes, vision s'enrichissant justement par ce partage, notamment selon le mécanisme d'idéation dont nous avons parlé. L'action distribuée met en lumière le fait que des individus travaillant en intercompréhension sont susceptibles d'avoir des représentations différentes au départ par rapport à une même situation entrepreneuriale ou action en devenir. Action permettant de réconcilier ces représentations différentes et d'enrichir la situation initiale. De ce point de vue, le fait de recourir à une logique causale ou à une logique effectuale, sinon d'improvisation, en situation, va dans ce sens (Smolka et al., 2016). À partir des travaux de Le Moigne (1990) et de Morin (1990), il est possible de qualifier ainsi l'agir entrepreneurial de complexe, c'est-à-dire se caractérisant par un grand nombre d'interactions, de représentations et d'incertitudes par rapport à son développement (Schmitt, Julien, Lachance, 2002).

Cela nécessite un travail de modélisation ou de scénarisation pour le chercheur. La conséquence en est qu'il n'est donc pas possible de prendre en compte tous les éléments liés à l'agir entrepreneurial. Se met alors en place un travail d'intelligibilité de l'agir, amenant l'entrepreneur à construire une représentation de l'agir à venir ou à l'imaginer de façon virtuelle pour la partager avec les autres acteurs de l'action située. Il s'agit d'orienter vers des représentations et des actions satisfaisantes - au sens du satisficing (Simon, 1996). C'est l'aspect écologique de l'agir entrepreneurial qui s'ajuste en fonction des environnements interne et externe. Ce mécanisme d'adaptation correspond au processus de conception déjà évoqué précédemment.

\section{CONCLUSION}

L'approche effectuale de Sarasvathy aura permis à la théorie entrepreneuriale de sortir des sentiers battus dans laquelle elle se trouvait depuis trop longtemps, en envisageant autrement l'entrepreneuriat. Elle a ainsi ouvert de nouvelles portes pour en comprendre la complexité, en permettant d'inclure explicitement, par exemple, l'intuition, l'anticipation, la flexibilité, les rétroactions et l'incertitude à court ou à moyen terme d'un bon nombre de projets entrepreneuriaux. Toutefois, le risque actuel que nous pourrions envisager dans ce sillage résiderait dans la dévalorisation des entrepreneurs qui se limiterait à une logique causale au détriment de ceux suivant une logique effectuale ou d'improvisation. Si l'apport de l'effectuation est indéniable pour la recherche en entrepreneuriat, il n'en demeure pas moins nécessaire de ne pas perdre de vue que cette logique s'est essentiellement construite en opposition à celle causale. Dans cet article, il nous semblait important de considérer avec d'autres que la logique causale et celle effectuale sont les deux faces d'une même pièce qu'il convient de considérer, en ajoutant, avec la tranche de cette pièce, la troisième dimension qu'est l'improvisation.

Finalement, nous avons proposé d'utiliser la notion, développée par Schmitt (2015a), d'agir entrepreneurial permettant de relier les trois logiques susmentionnées qui peuvent se retrouver à différents moments dans les pratiques des entrepreneurs et de leurs parties prenantes de même que l'écosystème. Le recours à l'agir entrepreneurial a aussi pour objectif de compléter le 
plus souvent un vide théorique au niveau de la recherche en entrepreneuriat. En se focalisant sur la dualité entre causation et effectuation, la recherche en entrepreneuriat s'est plus ou moins ou trop souvent inscrite dans le paradigme de la décision au détriment de l'action. Nous devons donc transformer notre façon de voir les choses pour développer des connaissances sur l'action et non plus uniquement sur l'entrepreneur ou sur le résultat de ses actions. Pour cela, nous nous situons dans une position métaphysique médiate par rapport à ces trois logiques en proposant de considérer l'agir comme élément commun entre elles, permettant de générer des connaissances nouvelles dans le domaine de l'entrepreneuriat.

Avec ce dernier point, nous souhaitons revenir sur les enseignements issus de notre réflexion, qui sont autant de pistes de recherche importantes à traiter, que nous inculquent ces trois approches quand nous les considérons comme plus d'une façon s'intégrant dans l'agir entrepreneurial.

S'intéresser spécifiquement à la place de l'agir entrepreneurial en entrepreneuriat nécessite enfin de s'ouvrir à d'autres domaines qui ont largement investigué l'agir humain en général et l'action en particulier. La recherche en entrepreneuriat se doit d'être pluridisciplinaire, voire transdisciplinaire pour faire bouger les frontières et sortir du tropisme rationnel cartésien dans lequel elle s'est construite avec Max Weber. Comme nous l'avons montré, par exemple, autour de la cognition située, la cognition distribuée ou encore de l'intentionnalité (Berglund, 2007), l'entrepreneuriat se devrait de mobiliser des concepts qui ne sont pas issus de son champ historique et disciplinaire. Néanmoins, cet excursionnisme scientifique, bien que stimulant, ne doit pas se faire de façon anarchique. Des pistes existent qu'il conviendrait d'explorer plus en aval, avant d'en ouvrir d'autres. Nous nous heurtons ici aux limites de la connaissance des chercheurs et dans une certaine mesure au manque d'intérêt que d'autres disciplines portent à l'entrepreneuriat, lequel apparaît souvent, par ailleurs, comme dilué dans une thématique plus grande.

Reste à mieux considérer le temps long et ainsi les diverses pratiques auxquelles les entrepreneurs recourent les unes à la suite des autres pour leur développement selon les circonstances de leur marché et les opportunités possibles. Ce qui conforte la conviction que toute analyse de l'agir entrepreneurial ne peut se comprendre réellement que d'une façon dynamique (Gielnik et al., 2014).

\section{RÉFÉRENCES}

Acs, Z.J. et Mueller, P. (2003). Employment effects of dynamics: Mice, gazelles and elephants, Small Business Economics, 30 (1), p. 85-100.

Acs, Z.J., Desai, S. et Klapper, L.F. (2008). « What does 'Entrepreneurship' Data Really Show? », Small Business Economics, vol. 31, n³, p. 265-281.

Ajzen, L. (1991). The theory of planned behavior. Organisational Behavior and Human Decision Processes, 50 (2), p. 179-211.

Alsos, G.A., Clausen, T.H., Hytty, U. et Solvoll, S. (2016). Entrepreneurial's social identity and the preference of causal and effectual behaviours in start-up processes. Entrepreneurship \& Regional Development, 28 (3-4), p. 234-258. 
Arend, R. J., Sarooghi, H. et Burkemper, A. (2015). Effectuation as ineffectual? Applying the 3E theory-assessment framework to a proposed new theory of entrepreneurship. Academy of Management Review, 40(4), p. 630-651.

Balachandra, L. (2019). The improvisational entrepreneur: Improvisation training in entrepreneurship education, Journal of Small Business Management, 57 (1), p. 60-77.

Baron, R.A. (2006). Opportunity recognition as pattern recognition: how entrepreneurs 'connect the dots' to identify new business opportunities. Academy of Management Perspective, 20 (1), p. 104-119.

Baron, R.A. et Markman, G.D. (2000). Beyond social capital: how social skills can enhance entrepreneurs' success. The Academy of Management Executive, 14 (14), p. 106-116.

Barrett, F.J. (1998). Creativity and improvisation in jazz and organizations: implications for organizational learning , Organization Science, numéro thématique sur la métaphore du jazz et l'organisation, 9 (5), p. 605-622.

Barrett, F.J. et Peplowski, K. (1998). Minimal structure within a song: an analysis of 'All of Me', Organization Science, 9 (5), p. 543-623.

Barringer, B.R., Jones, F.F. et Lewis, P.S. (1998). A qualitative study of the management practices of rapid-growth firms and how rapid-growth firms mistigate the managerial capacity problem, Journal of Developmental Entrepreneurship, 3 (2), p. 97-140.

Barwise, J. (1989). The Situation in Logic, Stanford, Center of the Study of Language Information.

Bauernschustera, S., Falcket, O. et Heblich, S. (2010). Social capital access and entrepreneurship, Journal of Economic Behavior \& Organization, 76 (30), p. 821-833.

Belliato, E., Champagne, C., Prim-allaz, I. et Séville, M. (2010). L'effet de levier de la confiance sur l'engagement dans l'hypercroissance durable des PME. Communication présentée au congrès de l'AIMS, date, Luxembourg.

Berger, P. et Luckmann, T. (1966). The Social Construction of Reality. New-York, Doubleday.

Berglund, H. (2007). «Researching entrepreneurship as lived experience », dans Neergaard, H. et Ulhøi, J. (dir.), Handbook of Qualitative Research Methods in Entrepreneurship, Cheltenham, Edward Elgar, p. 75-93.

Bergson, H. (1922). Durée et simultanéité. À propos de la théorie d'Einstein, Paris, PUQ.

Bird, B. et Schjoedt, L. (2009). Entrepreneurial behavior: Is nature, scope, recent research, and agenda for future research, Understanding the entrepreneurial mind International Studies in entrepreneurship), p. 327-358.

Blumer, H. (1969). Symbolic Interactionism. Berkeley, University of California Press.

Boyd, N.G. et Vozikis, G.S. (1994). The Influence of self-efficacy on the development of entrepreneurial intentions and actions. Entrepreneurship Theory and Practice, 18(4), p. 63-77.

Boudon, R. (1984). La place du désordre, Paaris, PUF.

Brown, S.L. et Eisenhardt, K.M. (1998). Competitive on the Edge. Strategy as Structured Chaos, Boston, Harvard Business School Press, chap. 2.

Bruton, G.D, Ahlstrom, D. et Li, H. (2010). Institutional theory and entrepreneurship. Where are we now and where do we need to move in the future. Entrepreneurship Theory and Practice 34(3), p. 421-440.

Bruyat, C. et Julien, P.A. (2001). Defining the field of research in entrepreneurship, Journal of Business Venturing, 16(2), p. 17-27.

Chandler, G.N., Detienne. D.R., Mckelvie, A. et Umfort, T.V. (2011). Causation and effectuation processes. A validation study. Journal of Business Venturing, 26(3), p. 375-390.

Chanut-Guieu, C. et Guieu, G. (2011). Stratégie et structuration des trajectoires d'hyper croissance des PME. Une étude de cas comparative. Management \& Avenir, n 43, p. 36-55.

Claveau, N., Perez, M. et Prim-Allaz, I. (2013). Vers une différenciation des trajectoires de forte croissance. Revue internationale PME, vol. 26, no 3-4, p. 117-139.

Cope, J. (2005). Toward a dynamic learning perspective of entrepreneurship, Entrepreneurship Theory and Practice, vol. 29, n 4, p. 373-397.

Corbett, A.C. et Katz, J.A. (2012). Entrepreneurial action, 14, Emerald Group Publishing Limited.

Cotta, A. (1980), La société ludique, Paris, Grasset. 
Davidsson, P. (2015). Entrepreneurial opportunities and the entrepreneurship nexus: A re-conceptualization. Journal of Business Venturing, 30(5), p. 674-695.

Davenport, T.H., DeLong, D.W. et Beers, M.C. (1998). Successful knowledge management projects. Sloan Management Review, 39 (2), p. 43-57.

Dew, N., Grichnik, D., Mayer-Haug, K., Read, S. et Brinckmann, J. (2015). Situated Entrepreneurial Cognition. International Journal of Management Reviews, 17(2), p. 143-164.

Dewey, J. (1938). Logic: the theory of inquiry. New York: Holt, Rinehart and Winston.

Dimov, D. (2007). Beyond the single-person, single-insight attribution in understanding entrepreneurial opportunity, Entrepreneurship Theory and Practice, 31(5), p. 713-731.

Dubar, C. (1996). La socialisation, construction des identités sociales et professionnelles, Paris, Armand Colin.

Duncan, R.B. (1972). Characteristics of organizational environments and perceived environmental uncertainties. Administrative Science Quarterly, vol. 17, n³, p. 313-327.

Estrin, S., Korosteleva, J. et Mickiewicz, T. (2013). Which institutions encourage entrepreneurial growth aspiration?, Journal of Business Venturing, vol. 28, n 4, p. 564-580.

Fisher, G. (2012). Effectuation, Causation, and Bricolage: A Behavioral Comparison of Emerging Theories in Entrepreneurship Research. Entrepreneurship: Theory and Practice, 36 (5), p. 1019-1051. Foerster, H. von (2000). Éthique et cybernétique de second ordre. Dans P. Watzlawick, et G. Nardone, (Eds.), Stratégie de la thérapie brève, Paris: Seuil, p. 60-76.

Frese, M. (2012). The psychological actions and entrepreneurial success: an action theory approach. Dans J. R. Baum, M. Frese and R. Baron, The Psychology of Entrepreneurship, New-York: Psychology Press, p. 151-184.

Fritsch, M. et Changoluisa, J. (2017). New business formation and the productivity of manufacturing incombents: Effects and méchanisms. Journal of Business Venturing, vol. 32 (2), p. 237-259.

Fritsch, M. et Wyrwich, M. (2017). The effect of entrepreneurship on economic development-an empirical analysis using regional entrepreneurship culture, Journal of Economic Geography. 17 (1), p. 157-189.

Gabrielson, J. et Politis, D. (2011). Career motives and entrepreneurial decision-making: Examining preferences for causal, and effectual logic in the early stages of new ventures. Small Business Economics, 36(3), p. 281-298.

Garfinkel, H. (1967). Studies in Ethnomethodology, Englewood Cliffs, Prentice-Hall.

Gartner, W.B. (1988). Who is an Entrepreneur? Is the wrong question. American Journal of Small Business 12(1): p. 11-22.

Geindre, S. et Dussuc, B. (2015). Capital social et recherche en PME. Revue Internationale PME, 28(1), p. 27-55.

Gielnik, M.M., Barabas. S., Frese, M., Namatovu-dwa, R., Scholz, F.A., Metzgger, J.R. et Walter, T. (2014). A temporal analysis of how entrepreneurial goal intentions, positive fantasies, and actions planning affect starting a new venture and when the effects wear off. Journal of Business Venturing, 29 (4), p. 755-772.

Gjerløv-juel. P. et Guenther, C. (2019). Early employment expansion and long-run survival examining employee turnover as a context factor. Journal of Business Venturing, vol. 34 (1), p. 80-102.

Glasersfeld, E. von, (1984). An introduction to radical constructivism. Dans P. Watzlawick (Ed.). The invented reality, Norton: New York, p. 17-40.

Godemer, A. (2002). PME en croissance: peut-on prévoir les seuils organisationnels ? Revue Internationale PME, 15(2), p. 39-63.

Goffman, E. (1974). Frame analysis: An essay on the organization of experience. London: Harper and Row.

Grégoire, D.A., Corbett, A.C. et McMullen, J.S. (2011). The cognitive perspective in entrepreneurship: an agenda for future research. Journal of Management Studies, 48(6), p. 1443-1477.

Grégoire, D.A., Cornelissen, J., Dimov, D. et Van Burg, E. (2015). The mind in the middle: Taking stock of affect and cognition research in entrepreneurship. International Journal of Management Reviews, 17(2), p. 125-142. 
Grimand, A. (2013). La gestion des ressources humaines dans les PME en hypercroissance : frein ou levier? Revue internationale PME : vol. 26, no 3-4, p. 89-115.

Grichnik, D. Smeja, A. et Welpe, I. (2010). The importance of being emotional: How do emotions affect entrepreneurial opportunity evaluation and exploitation, Journal of Economic Behavior \& Organization, 76 (1) p. 15-29.

Habermas, J. (1987). Théorie de l'agir communicationnel. Rationalité de l'agir et rationalisation de la société. Tome 1. Paris : Fayard.

Habermas, J. (1979). dans T.F. Geraerts (éd.), Actes du colloque de 1979 sur Rationality today/La rationalité aujourd'hui, Presses de l'Université d'Ottawa. https://doi.org/10.7202/203154ar

Hambrick, D.C. et Croizer, L.M. (1985). Stumblers and stars in the management of rapid growth, Journal of Business Venturing, 1 (1) p. 31-45.

Hatchuel, A. (2000). Quel horizon pour les sciences de gestion ? Vers une théorie de l'action collective. Dans A. David, A. Hatchuel, et R. Laufer (Eds.), Les nouvelles fondations des sciences de gestion, Paris: Vuibert/Fnege, p. 7-43.

Henrekson, M. et Johansson, D. (2010). Gazelles as job creators: a survey and interpretation of the evidence. Small Business Economics, vol. 35, no 2, p. 227-244.

Hindle, K. (2010). How community context affects entrepreneurial process: A diagnostic framework. Entrepreneurship and Regional Development, 22 (7-8), p. 599-647.

Hutchins, E. (1995). Cognition in the Wild. Cambridge, MA: MIT Press.

Jiang, Y. et Rüling, C.C. (2019). Opening the black Box of effectuation processes: Characteristics and dominant types. Entrepreneurship Theory and Practice, 43 (1). p. 171-2002.

Joas, H. (1996). The Creativity of Action. Chicago: Chicago University press.

Johannisson, B. (1995). Entrepreneurship networking in the Scandinavian context: Theoretical and empirical positioning. Entrepreneurship \& Regional Development 7 (2), p. 189-192.

Johannisson, B. (2011). Towards a practice theory of entrepreneuring. Small Business Economics, 36(2), p. 135-150.

Julien, P. A. (2001). Des gazelles, de la métaphore du jazz et d’autres choses. Comment gérer l'improvisation de façon cohérente Revue internationale PME, 14 (3-4), p. 129-162.

Julien, P.A. et Lachance, R. (2001). Dynamic regions and high-growth SME's: uncertainty, potential information and weak signal networks, Human System Management, 20 (3), p. 237-248.

Julien, P.A. (2003). Les PME à forte croissance : l'exemple de 17 gazelles dans 8 régions du Québec. Québec : Presses de l'Université du Québec.

Kerr, J. et Coviello, N. (2019). Weaving network theory into effectuation: A multi-level reconceptualization of effectual dynamics. Journal of Business Venturing. (à paraître).

Kim, W.C. et Mauborgne, R. (1997), Value innovation: the strategic logic of high growth, Harvard Business Review, 75 (1) p. 102-112.

Klein, P.J. (2008). Opportunity discovery, entrepreneurial action, and economic organization. Strategic Entrepreneurship Journal , 2 (3), p. 175-190.

Koenig, G. (2003). L'organisation dans une perspective interactionniste, in B. Vidaillet (dir.), Le sens de l'action, Paris : Vuibert, p. 15-34.

Krueger, N.F. et Carsrud, A.L. (1993). Entrepreneurial intentions: Applying theory of planned behavior, Entrepreneurship and Regional Development, 5 (3), p. 315-330.

Lave, J. (1988). Cognition in practice, Cambridge University Press, Cambridge.

Le Moigne, J.-L. (1990). La modélisation des systèmes complexes. Paris: Dunod.

Lerner, D.A. (2016). Beheviorial disinhibition and nascent venturing: Rlevanc an initia effects on potential resources providers. Journal of Business Venturing, 31 (3), p. 234-252.

Lerner, D.A., Hunt, R.A. et Dimov, D. (2018). Action ! Moving beyond the intendedly-rational logics of entrepreneurship. Journal of Business Venturing, 33 (1), p. 52-69.

Lévesque, M. et Stephan, U. (2019). It's time we talk about time in entrepreneurship. Entrepreneurship: Theory and Practice, éditorial, là paraitre).

Liao, J., Welsch, H. et Stoica, M. (2008). Environmental turbulence and scanning behavior: the moderating effects of organizational maturity. Journal of Small Business Strategy, 19(1), p. 15-31. 
Lorino, P. et Teullier, R. (2005). Des connaissances à l'organisation par l'agir collectif. Dans R. Teulier et P. Lorino, Entre connaissance et organisation : l'activité collective, Paris : La Découverte, p. 11-20.

Marchesnay, M. (2002). Pour une approche entrepreneuriale de la dynamique ressourcescompétences. Les cahiers de l'ERFI, no 22, Université de Montpellier.

Marchesnay, M. et Julien, P.A. «Small business as space of transaction », Entrepreneurship \& Regional Development, vol. 2, n² 2, 1990, p. 267-277.

McKelvie, A., Haynie, J.M. et Gustavsson, V. (2011). Unpacking the uncertainty construct: implications of entrepreneurial action, Journal of Business Venturing, 26(2), p. 273-292.

McMullen, J.S. et Shepherd, D.A. (2006). Entrepreneurial action and the role of uncertainty in the theory of the entrepreneur. Academy of Management Review, 31 (1), p. 132-152.

Miles, M. et Snow, C.C. (1978). Organizational Strategy, Structure and Process, Mc Graw Hill, New York.

Milliken, F.J. (1987). Three types of perceived uncertainty about environment: State, effect. an the response uncertainty. Academy of Management Review, 12 (1), p. 133-143.

Mitusch, K. et Schimke, A. (2011). High-Growth Companies. Rapport horizontal final, $n^{\circ} 5$, Project $n^{\circ} 4$, Europa Innova.

Moreno, A.M. et Casillas, J.C. (2007). High-growth SMEs versus non-high-growth SMEs: a discriminant analysis. Entrepreneurhip and Regional Development, vol. 19, no 1, p. 69-87.

Morin, E. (1990). Introduction à la pensée complexe, Paris, ESF éditeur.

Moroz, P. W. et Hindle K. (2012). Entrepreneurship as a process : toward harmonizing multiple perspectives. Entrepreneurship Theory and Practice, 36 (4), p. 781-818.

Murnieks, C.Y., Mosakowski E. et Cardon, M.S. (2014). Pathways of passion, identity centrality, passion, and behavior among entrepreneurs. Journal of Management, 40 (6), p. 1583-1606.

Ngoasong, M.Z. et Kimbu, A.N. (2019). The slow process of high growth in women-owned businesses in a resource-scarce context, Journal of Small Business Management, 57 (1), p. 40-58.

OCDE. (2002). Les PME à forte croissance et l'emploi. Paris, OCDE.

Parsons, T. (1937). The Structure of Social Action, Glencow (ill.), Open Library.

Penrose, E.T. (1959). Français The Theory of the Growth of the Firm, Oxford, Oxford University Press.

Perry, J.T., Chandler, G.N. et Markova, G. (2011). Entrepreneurial effectuation: a review and suggestions for future research, Entrepreneurship Theory and Practice, 36 (4), p. 837-861.

Rannikko, H., Tornikoaki, E.T., Isaksson, A. et Löfsten, H. (2019). Survival and growth patterns among new technology-based firms: Empirical study of cohort 2006 in Sweden, Journal of Small Business Management, 57 (2), p. 460-657.

Razafindrazaka, T. et Julien, P.A. (2017). Le rôle du capital social dans la modernisation d'un écosystème entrepreneurial traditionnel : le cas de la région de Vakinankaratra, Revue internationale $P M E$, vol. 30, nos 3-4, p. 99-126.

Read, S. et Sarasvathy, S. (2005). Knowing what to do and doing what you know: effectuation as a form of entrepreneurial expertise, Journal of Private Equity, 9 (1), p. 45-62.

Sarasvathy, S. (2001). Causation and Effectuation: Toward a Theoretical Shift from Economic Inevitability to Entrepreneurial Contingency. Academy of Management Review, 26 (2), p. 243-263.

Saravasthy, S. (2003). Entrepreneurship as a science of the artificial. Journal of Economic Psychology, 24 (2003), p. 203-220.

Sarasvathy, S. (2008). Designing organizations that design environments: Lessons from entrepreneurial expertise, Organization Studies, 29 (3), p. 331-350.

Sarasvathy, S. et Venkataraman, S. (2011). Entrepreneurship as method: Open questions for an entrepreneurial future, Entrepreneurship Theory and Practice, 35 (1), p. 113-135.

Schmitt, C., Julien, P.A. et Lachance, R. Pour une lecture des problèmes complexes en PME : approche conceptuelle et expérimentation, dans Revue Internationale PME, vol. 15, No2, p. 35-62.

Schmitt, C. (2009). Les situations entrepreneuriales: proposition d'une nouvelle grille d'analyse pour aborder le phénomène entrepreneurial, Revue économie et sociale, no 3, p. 11-25.

Schmitt, C. (2015a). L'agir entrepreneurial. Repenser l'action des entrepreneurs, Presses de l'Université du Québec, Québec. 
Schmitt, C. (2015b). La place de l'action dans la recherche en entrepreneuriat: pour le développement d'un agir entrepreneurial. Projectics / Proyéctica / Projectique, 15(3), p. 113-128.

Schmitt, C. (2017a). La fabrique de l'entrepreneuriat, Dunod, Paris.

Schmitt, C. (2017b). Quel paradigme pour la recherche en entrepreneuriat ? Du paradigme de la décision au paradigme de l'agir entrepreneurial, numéro thématique 80 ans de la revue, Gestion 2000, vol. 34, no. 5, p. 249-256.

Schmitt, C. et Gregoire, D. (2018). La Cognition entrepreneuriale. Enjeux et perspectives pour la recherche en entrepreneuriat, Revue de l'Entrepreneuriat, Vol. 18, n¹, p.7-22.

Shackle, G.L.S. (1979). Imagination and the Nature of Choice. Édimbourg, Edinburgh University Press.

Shapero, A. (1975). The displaced, uncomfortable entrepreneur, Psychology Today, 9 (6), p. 83-88.

Shapero, A. et Sokol, L. (1982). The Social Dimensions of Entrepreneurship. Dans C. Kent, et al. (Eds) The Encyclopedia of Entrepreneurship. Englewood Cliffs, NJ: Prentice-Hall, p. 72-90.

Simon, H. A. (1996). The sciences of the artificial. Cambridge: MIT Press.

Smallbone, D., Leig, R. et North, D. (1995). “The characteristics and strategies of high growth SMEs." International Journal of Entrepreneurial Behavior \& Research, vol. 1, no 3, p. 44-62.

Smith, K.G. et Di Gregorio, D. (2002). Bisociation, discovery, and the role of entrepreneurial action. Dans M.A. Smith, R.D. Ireland, S.M. Camp et D.L. Sexton. Strategic Entrepreneurship, Hoboken, Wiley-Blackwel.

Smolka, K.M., Verheul, I., Burmeister-lamp, K. et Heugens, P. (2016). get-it together! synergistic effect of causal and effectual decision-making logic on venture performance, Entrepreneurship Theory \& Practice, 42 (4), p. 571-604.

Spigel, B. (2015). The relational organization of entrepreneurial ecosystems, Entrepreneurship Theory \& Practice, 41 (1), p. 49-72.

Steyaert, C. (2007). 'Entrepreneuring' as a conceptual attractor? A review of process theories in 20 years of entrepreneurshup studies. Entrepreneurship \& Regional Development, 19, no ..., p. 453-477.

Steyaert, C., et Landström, H. (2011). Enacting entrepreneurship research in a pioneering, provocative and participative way: On the work of Bengt Johannisson. Small Business Economics, 36, no 2, p. 123-134.

Stinchfield, B.T., Nelson, R.E. et Wood, M.S. (2012). Learning from Levi-Strauss' legacy: Art, Craft, Engineering, Bricolage, and Brokerage in entrepreneurship. Entrepreneurship Theory \& Practice, p. 37 (4).

St-Jean, E., Julien, P.A. et Audet, J. (2008). Factors of high-growth SMEs, Journal of Enterprising Culture, vol. 16, no 2, p. 161-188.

Suchman, L.A. (1987). Plans and situated actions: The problem of human-machine communications. Cambridge, Cambridge University Press.

Tang, J., Tang, Z. et Katz, J.A. (2014). Proactiveness stakeholder-firm power difference and product safety and quality of Chinese SMEs. Entrepreneurship Theory \& Practice, 41 (5), p. 1129-1157.

Theureau, J. (1992). Le cours d'action: analyse sémiologique. Essai d'une anthropologie cognitive située, Berne, Peter Lang.

Tsoukas, H. (2005). Complex Knowledge, New York, Oxford University Press.

Vaghely, I.P. et Julien, P.A. (2010). Are opportunities recognized or constructed? An information perspective on entrepreneurial opportunity recognition, Journal of Business Venturing, 25, no 1 , p. 73-86.

Venkataraman, S., Sarasvathy, S., Dew, N. et Forster, W. (2012). Whither the promise? Moving forward with entrepreneurship as a science of the artificial. Academy of Management Review, 37 (1), p. 21-33.

Vermersch, P. (1994). L'entretien d'explicitation, Issy-les-Moulineaux, ESF éditeur.

Vygotsky, L.S. (1978). Mind in society: The development of higher psychological process. Cambridge, Harvard University Press.

Watson, T.J. (2013). Entrepreneurship in action. Bridging together the individual, organization and institutional dimension of entrepreneurial action, Entrepreneurship and Regional Development, 25 (5-6), p. 177-188. 
Watzlawick, P. (2000). La construction des réalités cliniques. Dans P. Watzlawick et G. Narbonne (coll.), Stratégie de la thérapie brève, Paris, Seuil, p. 19-33.

Weick, K. E. (1999). Theory Construction as Disciplined Reflexivity: Tradeoffs in the 90's. Academy of Management Review, 24 (4), p. 797-806.

Welter, F. (2011). Contextualizing entrepreneurship, conceptual challenges and ways forward, Entrepreneurship Theory and Practice, 33(1), p. 165-184.

Welter, C. et Kim, S. (2018). Effectuation under risk and uncertaint: A simulation model. Journal of Business Venturing, 33 (1), p. 100-116.

Wernerfeld, B. (1995). The resource-based view of the firm: Ten years after, Strategy Management Journal, 16 (2), p. 171-174.

Wiklund, J., Davidsson, P. et Delmar, F. (2003). What do they think and feel about growth? An expectancy-value approach to small business managers' attitudes toward growth, Entrepreneurship Theory and Practice, 27 (3), p. 247-269.

Wood, M.W., Williams, D.W. et Grover, W. (2017). past as prologUe: entrepreneurial inaction decisions and subsequent action judgment. Journal of Business Venturing, 32 (2), p. 107-127.

Christophe SCHMITT est Vice-Président de l'Université de Lorraine. Il est Professeur des Universités à l'IAE de Metz (France). Il y est Titulaire de la Chaire «Entreprendre » et responsable du Pôle entrepreneuriat étudiant de Lorraine (PeeL). Il est également Professeur associé à la Louvain School of Management (Belgique) et à la Haute Ecole de Gestion de Fribourg (Suisse). Ses articles et ouvrages portent sur le développement d'une théorie de l'action entrepreneuriale. Il est l'auteur de « La fabrique de l'entrepreneuriat » paru chez Dunod.

Pierre-André JULIEN est professeur émérite. Il a publié 24 ouvrages et 108 articles sur l'économie des PME et l'entrepreneuriat. Il a été le premier expertconseil dans ce domaine à l'OCDE de 1989 à 2010. Il est co-fondateur de la Revue internationale PME et de l'AIREPME. 